\title{
The Diagnosis and Therapy of Retained Stones and Residual Nasobiliary Drainage Tube in the Common Bile Duct via Endoscope: Report of a Rare Complication
}

\author{
Zhiyong Wang ${ }^{1, *}$, Sensen Tang ${ }^{1}$, Jinlong $\mathrm{Fu}^{1}$, Haigang Shen ${ }^{2}$, Mingrong $\mathrm{Hu}^{2}$, Chenggang $\mathrm{Xu}^{2}$, Xinsuo Zhuang ${ }^{2}$, \\ Yadong $\mathrm{Liu}^{2}$, Jianliang $\mathrm{Wu}^{2}$ \\ ${ }^{1}$ Hangzhou normal university medical college, Zhejiang, China \\ ${ }^{2}$ The affiliated hospital of Hangzhou normal university, Zhejiang, China \\ *Corresponding author: zywang157@sina.com
}

Received May 20, 2015; Revised June 03, 2015; Accepted June 16, 2015

\begin{abstract}
Introdution: Foreign body in biliary tract is a rare iatrogenic complication, and the patient usually has a medical history of surgeries in biliary system. The foreign body in biliary tract blocks the biliary tract and induces acute cholangitis and cholecystitis, some become cores and form the common duct stones. The upper abdominal pain can occur repeatedly accompanied by fever, icterrus, nausea and vomiting, and the blood test and liver function turn out abnormal. In combination with medical history, clinical manifestation, type B ultrasound, CT and MRCP, the diagnosis can be confirmed. Generally speaking, surgical treatment is considered as the principle. With the improvement in ERCP technology and endoscopic accessories, the minimally invasive interventional therapy has been further applied. Case presentation: A 72-year old patient, who experienced upper abdominal pain during the hospitalization in our hospital and the diagnosis was confirmed to be cholecystolithiasis and multiple stones in common bile duct after type B ultrasound and CT. The patient underwent ERCP, EST, biliary tract calculus removal and LC surgery. After discharge, the patient intermittently experienced upper abdominal discomfort and dull abdominal pain without fever and icterus. Type B ultrasound and CT: the thin stripped shaped tubular substance and calculus shadows were observed inside common bile duct. Then the patient underwent ERCP, which indicated the residual end of nasobiliary drainage tube of intestinal cavity of descending part of duedenum, the length was about 6 $\mathrm{cm}$, and it was removed using a calculus dislodger. It was difficult to target the residual end of the nasobiliary drainage tube in the common bile duct using a calculus dislodger, so the calculus dislodger was replaced by stoneremoving Foley's tube and finally an about $8 \mathrm{~cm}$ long residual end of nasobiliary drainage tube was taken out. Conclusion: There are only a few case reports in the literature describing the iatrogenic complications of retained stones and residual nasobiliary drainage in the common bile duct after having ERCP. We describe the diagnosis and therapy of the complications by endoscopy to alert clinician.
\end{abstract}

Keywords: retained stones, residual nasobiliary drainage tube, common bile duct, diagnosis, therapy

Cite This Article: Zhiyong Wang, Sensen Tang, Jinlong Fu, Haigang Shen, Mingrong Hu, Chenggang Xu, Xinsuo Zhuang, Yadong Liu, and Jianliang Wu, "The Diagnosis and Therapy of Retained Stones and Residual Nasobiliary Drainage Tube in the Common Bile Duct via Endoscope: Report of a Rare Complication.” American Journal of Medical Case Reports, vol. 3, no. 7 (2015): 222-224. doi: 10.12691/ajmcr-3-7-11.

\section{Case Report}

A 72-year old man was admitted on July 22, 2014 due to "slurred speech accompanied unstable gait for 12 hours". During in the hospital, abdominal ultrasound examination revealed chronic cholecystitis accompanied by cholecystolithiasis, the common bile duct was $9 \mathrm{~mm}$ in diameter, and stone at the upper segment was suspected. The liver was noted via CT to contain multiple round low density images, the largest was sized about $32.8 \mathrm{~mm} *$ $29.6 \mathrm{~mm}$. In addition, CT prompted a thickened gallbladder wall and high density shadow of cholecyst plus choledoch. Biochemical assays showed the serum bilirubin and alkaline phosphatase levels to be slightly elevated, but the serum liver transaminase levels were approximately normal. It was not long before the patient experienced an attack of severely epigastric pain. In consideration of the patient's symptoms and examinations, on Aug. 8, the patient underwent ERCP + EST + columnar balloon dilation + removing common bile duct calcui with reticular basket + ENBD, 8 stones with a diameter of $0.5 * 0.6 \mathrm{~cm}$ were removed from the common bile duct (Figure 1-Figure 2); on Aug. 28, the patient underwent laparoscopic cholecystectomy; on Sep. 2, the patient had the nasobiliary drainage tube pulled out; duodenal papillary pathology: chronic papillary inflammation accompanied by proliferation of granulation tissue, and mild dysplasia of local glandular organs. The patient was hospitalized for 45 days. After discharge, the patient 
intermittently experienced upper abdominal discomfort and dull abdominal pain without fever and icterus. Type B ultrasound: mild dilation was seen in the common bile duct, and long strip-shaped high density shadows were seen in the common bile duct. CT: Dilation and stone shadow were seen, and high density long strip shaped shadows were seen in intrahepatic and extrahepatic ducts. On Mar. 19, 2015, the patient underwent ERCP again, which indicated the residual end of nasobiliary drainage tube of intestinal cavity of descending part of duedenum (Figure 3), the length was about $6 \mathrm{~cm}$, it was removed using a calculus dislodger (Figure 4), the duodenal papilla presented the incision type, radiography indicated that thin long strip-shaped tubular substances were seen in the common bile duct and occupied the intrahepatic and extrahepatic ducts (Figure 5), and a calculus dislodger and snare were used to remove the tubular foreing bodies repeatedly but ended in vain. Then they were replaced by stone-removing Foley's tube. The tube was inserted into the hepatic hilum, and $2.5 \mathrm{ml}$ of gas was injected into the stone-removing balloon. After the inflation of the balloon, the balloon catheter was slowly pulled out and the same time the tubular foreign bodies as a whole were shifted to the inferior part of the common bile duct to the orifice of papilla, but they could not be pulled out of the papilla. Then the foreign body forceps was utilized to clamp the lower end of the foreign body, then slowly take it out and place it into the nasobiliary drainage tube. It turned out that the tubular substance was the residual end of the nasobiliary drainage tube, the length was about $8 \mathrm{~cm}$, the surface was black, folds were noted at the lateral aperture of the middle segment and were about to rupture, and after measurement, the total length of the residual end of nasobiliary drainage tubes was $14 \mathrm{~cm}$ (Figure 7). 1 week after operation, no foreign residue was seen in the radiography of nasobiliary drainage tube. After 1-week hospitalization, the patient was discharged.

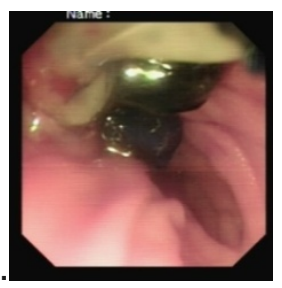

Figure 1. First ERCP stone removal

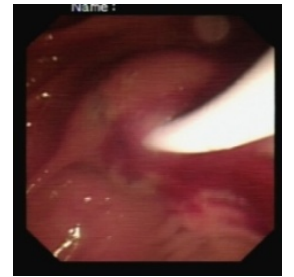

Figure 2. First ENBD

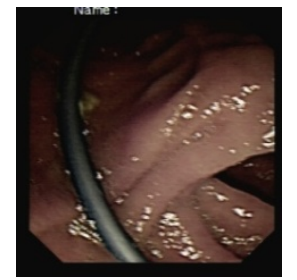

Figure 3. Residual ends of nasobiliary drainage tube at the intestinal cavity

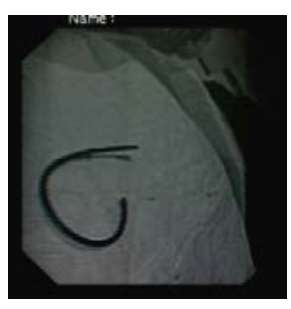

Figure 4. ERCP indicated the foreign body in biliary tract

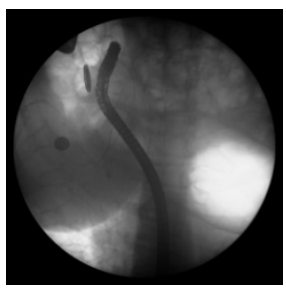

Figure 5. Removing residual ends of nasobiliary drainage tube

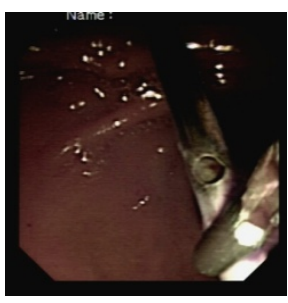

Figure 6. Removing foreign body in biliary tract using a foreign body forceps

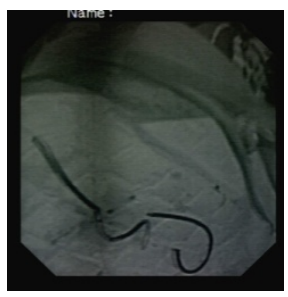

Figure 7. Removing residual ends of nasobiliary drainage tube

\section{Discussion}

To the best of our knowledge based on a literatureassisted search, there is rare report of foreign bodies of biliary tract in clinical medicine. The causes are as follows: (1) it is an iatrogenic complication, the surgical hemostatic clamp has been widely used, and it is generally considered to be very safe. However, after laparoscopic cholecystectomy, the migration of titanium clip to the common bile duct was observed and generally considered to be the bile duct injury, the improper placement of titanium clip, the sub-clinical bile leakage and infection facilitated the migration of titanium clip. Some investigators reported that, the suturing materials such as silk, chromicized catgut, parasites and foreign bodies such as fish bones became the cores of the common duct stones $[1,2,3]$; the common complications after ERCP surgery include acute pancreatitis, cholangeitis, intestinal perforation and bleeding etc., and it is rarely reported that the ERCP surgery induces foreign bodies in biliary tract, such as Zebra guide wires and titanium clips [2]; (2). The foreign body that retrogradely enters the biliary tract via duodenal papilla: as is reported by Dias R. et al [4], a 5year old boy accidentally took a metal needle and it was 
found after surgical exploration to be within the duodenal common bile duct and eventually taken out through the duodenal ampulla. This is a report on an ingested metal needle at the common bile duct, and it is the first reported case among the literatures all over the world. Yao Minsheng et al. reported [5] that the patient was a farmer and used common bamboo tools for cooking. The bamboo stick was accidentally ingested when eating, the stick was $4.5 \mathrm{~cm}$ long, it retrogradely entered the common bile duct, and the case was really rare. (3). Other unknown reasons. Such foreign bodies are taken out through reticular basket under ERCP and surgical operation. The patient underwent ERCP, EST, biliary tract calculus removal and LC surgery. After 24 hours operation, the patient had the nasobiliary drainage tube pulled out. After discharge, the patient intermittently experienced upper abdominal discomfort and dull abdominal pain without fever and icterus. Type B ultrasound and CT: the thin stripped shaped tubular substances were observed inside common bile duct. The patient underwent ERCP again, it was difficult to use a calculus dislodger to take out the foreign body, then stone-removing Foley's tube was used instead and eventually about $8 \mathrm{~cm}$ long residual end of the nasobiliary drainage tube was taken out, and this method is worth borrowing. After analysis, the reason is the rupture when the nasobiliary drainage tube was pulled out. Experience and lession: first, pay special attention to the quality of the nasobiliary drainage tube; when the nasobiliary drainage tube is inserted, avoid the formation of folds in the biliary tract; after the nasobiliary drainage tube is pulled out, the intactness of the tube must be inspected; it is inappropriate to place the nasobiliary drainage tube in the biliary tract for too long.

\section{References}

[1] Gonzalez FJ, Dominguez E, Lede A, Jose P, Miguel P. Migration of vessel clip into the common bile duct and late formation of choledocholithiasis after laparoscopic cholecystectomy. Am J Surg. 2011; 202(4): 41-3.

[2] Chong VH, Chong CF. Biliary complications secondary to postcholecy stectomy clip migration: a review of 69 cases. J Gastrointest Surg. 2010; 14(4): 688-96.

[3] Kaji H, Asano N, Tamura H, Yuh I. Common bile duct stone caused by a fish bone: report of a case. Surg Today. 2004; 34(3): 268-71.

[4] Dias R, Dharmaratne P. Ingested foreign body in the common bile duct. J Indian Assoc Pediatr Surg. 2012; 17(1): 31-2.

[5] Yinsheng Yao, Huiqun Li, Yulan Xiong. The foreign body of bamboo stick in common bile duct: a case of report. Chin J Gen Surg. 2000; 15(8):485. 